\title{
ASYMPTOTIC BEHAVIOUR FOR AN EQUATION OF SUPERSLOW DIFFUSION. THE CAUCHY PROBLEM
}

\author{
By
}

Victor A. Galaktionov

and

Juan L. Vazquez

IMA Preprint Series \# 841

August 1991 


\title{
ASYMPTOTIC BEHAVIOUR FOR AN EQUATION OF SUPERSLOW DIFFUSION. THE CAUCHY PROBLEM
}

\author{
VICTOR A. GALAKTIONOV* AND JUAN L. VAZQUEZ**
}

\begin{abstract}
We investigate the asymptotic behaviour as $t \rightarrow \infty$ of the nonnegative weak solution to the Cauchy problem for the equation of superslow diffusion

$$
u_{t}=\left(e^{-1 / u}\right)_{x x} \text { for } x \in \mathbb{R}, t>0
$$

with nonnegative initial function $u_{0} \in L^{\infty}(\mathbb{R}) \cap L^{1}(\mathbb{R}), u_{0} \not \equiv 0$. We prove that asymptotic separation of variables takes place if we make the change of variables $v=e^{-1 / u}$ and $\eta=x / \log t$. The precise result says that as $t \rightarrow \infty$

$$
t v(\eta \log t, t) \rightarrow \frac{1}{2}\left(a^{2}-\eta^{2}\right)_{+}
$$

and the convergence is uniform in $\eta \in \mathbb{R}$. The constant $a>0$ is exactly one half of the initial energy: $a=\frac{1}{2} \int u_{0}(x) d x>0$. This implies that $u$ evolves for large $t$ towards a mesa-like profile of height $1 /(\log t)$ and width $=2 a \log t$.
\end{abstract}

Key words. Nonlinear diffusion equation, asymptotic behaviour, explicit solutions

AMS(MOS) subject classifications. $35 \mathrm{~K} 55,35 \mathrm{~K} 65,34 \mathrm{E} 10$

Introduction. In this paper we investigate the asymptotic behaviour of the solution to the Cauchy problem for the equation

$$
u_{t}=(\phi(u))_{x x} \text { in } Q=\mathbb{R} \times(0, \infty),
$$

with diffusion law of the exponential type:

$$
\phi(u)=\epsilon^{-1 / u} \text { for } u>0, \quad \phi(0)=0,
$$

and the initial condition

$$
u(x, 0)=u_{0}(x) \text { in } \mathbb{R} .
$$

The initial function $u_{0}$ satisfics 
Equation (0.1), (0.2) for $u \geq 0$ is an example of equation of superslow diffusion, so-called because the diffusivity $\phi^{\prime}(u)=e^{-1 / u} u^{-2}$ vanishes at $u=0$ faster than any power of $u$. A large class of such equations has been studied in $[F]$. Existence and uniqueness of a continuous nonnegative weak solution of $(0.1)-(0.3)$ is well-known. The solution is smooth at any point of positiveness. See a full list of references given in [K].

In order to state our main result we introduce the change of variable $v=\phi(u)$, i.e.,

$$
v(x, t)=e^{-1 / u(x, t)} .
$$

Then, $0 \leq v(x, t)<1$ in $Q$, and $v(x, t)$ solves the quasilinear equation

$$
v_{t}=v(\log v)^{2} v_{x x} \text { in } Q .
$$

The asymptotic behaviour of $v(x, t)$ is exactly described by the following result.

TheOREM 1. Under hypotheses (0.4) we have

$$
\lim _{t \rightarrow \infty} t v(\eta(\log t), t)=F_{a}(\eta) \equiv \frac{1}{2}\left(a^{2}-\eta^{2}\right)_{+}
$$

uniformly for $\eta \in \mathbb{R}$, where $a$ is one half of the initial energy:

$$
a=\frac{1}{2} \int u_{0}(x) d x>0 .
$$

If we translate this result $(0.7)$ to the function $u(x, t)$ by means of the inverse transformation

$$
u(x, t)=-\frac{1}{\log v(x, t)},
$$

we get the asymptotic formula:

$$
\lim _{t \rightarrow \infty}(\log t) u(\eta(\log t), t)=1
$$

uniformly in any set $\{|\eta| \leq c\}$, where $c=$ const $\in(0, a)$, while for $|\eta| \geq a$ we have

$$
\lim _{t \rightarrow \infty}(\log t) u(\eta(\log t), t)=0 .
$$

Thus, in terms of the initial variable $u(x, t)$ we observe a mesa-like behaviour. Notice that the only parameter which appears in the formulas is the normalized length of the support of $u$, namely $2 a$. This parameter is easily calculated from the law of conservation of mass $\int u(x, t) d x=$ const, since for large $t$ it follows from $(0.10),(0.11)$ that $\int u(x, t) d x \approx 2 a$. 
Hence, $\left\|u_{0}\right\|_{1}=2 a$. Any further information about the asymptotic spatial structure of the solution as $t \rightarrow \infty$ is lost in the $u$ variable.

It is interesting to compare Theorem 1 with the asymptotic behaviour of the solution to the initial-boundary value problem for equation $(0.1),(0.2)$ in a bounded domain. In collaboration with $\mathrm{R}$. Kersner we have proved in [GKV] that for the one-dimensional problem posed in $(-l, l) \times(0, \infty)$, where $l>0$ is a fixed constant, with conditions

$$
\begin{gathered}
u(x, 0)=u_{0}(x) \text { in }(-l, l), \quad u_{0} \in L^{\infty}, u_{0} \geq 0, u_{0} \not \equiv 0, \\
u=0 \text { for } x= \pm l, t>0
\end{gathered}
$$

the following behaviour holds uniformly in $(-l, l)$ :

$$
\lim _{t \rightarrow \infty} t(\log t)^{2} v(x, t) \rightarrow F_{l}(x)
$$

Two differences appear. Firstly, the rate of decay is $t^{-1}(\log t)^{-2}$ and not $t^{-1}$ as in (0.7). Secondly, the particular asymptotic profile is determined by the length of domain and not by the initial function. However, it is remarkable that the family of asymptotic profiles is the same for the Cauchy and the initial-boundary value problems. This was not true for the porous medium equation $u_{t}=\left(u^{m}\right)_{x x}$ for a fixed $0<m<\infty$, see references in [K] , $[\mathrm{GV}]$.

We also obtain a precise result on the asymptotic behaviour of interfaces of every compactly supported solution to the Cauchy problem (0.1)-(0.3).

TheOREM 2. Assume that (0.4) holds and also that $u_{0}$ has a compact support. Then as $t \rightarrow \infty$

$$
\begin{aligned}
& s_{+}(t) \equiv \sup \{x \in \mathbb{R}: u(x, t)>0\}=a \cdot \log t+O(1), \\
& s_{-}(t) \equiv \inf \{x \in \mathbb{R}: u(x, t)>0\}=-a \cdot \log t+O(1) .
\end{aligned}
$$

After stating our main result, let us make some comments before proceeding with the proofs. Thus, in order to understand the appearance of the asymptotic profile $F_{a}(\eta)$ it is convenient to view our result in terms of the rescaled function $\theta$ corresponding to our asymptotic formula (0.7), which is defined by

$$
\theta(\eta, \tau)=(2+t) v(\eta \log (2+t), t)
$$

where $\tau=\log (2+t):[0, \infty) \rightarrow\left[\tau_{0}, \infty\right), \tau_{0}=\log 2$ (the number 2 plays no special role; any number $T>0$ would do). Then $\theta(\eta, \tau)$ solves the Cauchy problem

$$
\theta_{\tau}=\mathcal{C}(\theta, \tau) \equiv \mathcal{A}(\theta)+\frac{1}{\tau}\left(\theta_{\eta} \eta-2 \theta(\log \theta) \theta_{\eta \eta}\right)+\frac{1}{\tau^{2}} \theta(\log \theta)^{2} \theta_{\eta \eta}
$$


in $\mathbb{R} \times\left(\tau_{0}, \infty\right)$, with initial condition

$$
\theta\left(\eta, \tau_{0}\right)=\theta_{0}(\eta) \equiv 2 \exp \left\{-1 / u_{0}(\eta \log 2)\right\}
$$

The autonomous part of the operator in the right-hand side of $(0.16)$ has the form

$$
\mathcal{A}(\theta)=\theta \theta_{\eta \eta}+\theta
$$

It is easily seen that the functions $F_{a}(\eta)$ given in $(0.7)$ are precisely the radially symmetric and nonnegative weak solutions of the stationary equation $\mathcal{A}(\theta)=0$ which are monotone nonincreasing in $|\eta|$. Therefore Theorem 1 amounts to proving the convergence of the solution $\theta(\eta, \tau)$ as $\tau \rightarrow \infty$ to the corresponding stationary solution

$$
\mathcal{A}(F)=0 \text { in } \mathbb{R}, \quad F \geq 0, \quad F=F(|\eta|),
$$

which is uniquely determined by the total mass of the initial function, see $(0.8)$.

It is interesting to note that the function

$$
V(x, t)=t^{-1} F_{a}(x / \log t)
$$

describing by (0.7) the asymptotic behaviour of the solution $v(x, t)$ as $t \rightarrow \infty$, satisfies the nonautonomous quasilinear parabolic equation

$$
v_{t}=(\log t)^{2} v v_{x x}-\frac{x}{t \log t} v_{x}
$$

which looks quite different from (0.6). Thus, (0.20) is only an approximate self-similar solution of equation (0.6).

As for equation $(0.1),(0.2)$, the function $U(x, t)=(-\log V(x, t))^{-1}$, which is an approximate self-similar solution to $(0.1),(0.2)$, is in fact an explicit self-similar solution of the quasilinear equation

$$
u_{t}=(\log t)^{2} u^{2}\left(e^{-\frac{1}{u}}\right)_{x x}-\frac{x}{t \log t} u_{x}
$$

We finally remark that a simple rescaling allows to extend our result to the more general equation $u_{t}=(\phi(u))_{x x}$ with $\phi(u)=a e^{-b / u}, a, b>0$.

1. Preliminaries. Explicit Solution. The weak solution to the problem $(0.1)-(0.3)$ is a continuous nonnegative function which is smooth at any point where $u>0$ and has a continuous heat flux $-\left(e^{-1 / u}\right)_{x}$ on interfaces $\{u=0\}$; see for details the list of references 
in the review $[\mathrm{K}]$. We also note that for the solution of nonlinear equations of the type (0.1) the law of conservation of mass holds, i.e., if the initial mass is finite

$$
\int u_{0}(x) d x=E_{0}>0
$$

then

$$
\int u(x, t) d x=E_{0} \text { for any } t>0 \text {. }
$$

In view of $(0.5)$, this implies that

$$
\int_{-\infty}^{\infty}\left(-\frac{d x}{\log v(x, t)}\right)=\int_{-\infty}^{\infty}\left(-\frac{d x}{\log v_{0}(x)}\right)=E_{0} \text { for } t \geq 0
$$

The proof of our result is based on a careful use of a family of explicit solutions which can be found in $[\mathrm{BMFF}],[\mathrm{Ki}]$ :

$$
v_{*}(x, t ; c)=\frac{1}{2 t}\left(c^{2}-w^{2}\right)_{+}
$$

where $c>0$ is a fixed arbitrary constant and the function $w=w(x, t ; c) \in[0, c)$ is determined by the equation

$$
|x|=\Phi(w, c) \equiv(2+\log (2 t)) w+(c-w) \log (c-w)-(c+w) \log (c+w)
$$

Since the function $\Phi(w, c)$ in the right-hand side satisfies

$$
\Phi_{w}^{\prime}=\log (2 t)-\log \left(c^{2}-w^{2}\right) \geq 0
$$

for fixed $c>0$ and $t \geq c^{2} / 2$, equation (1.5) uniquely determines the function $w(x, t ; c) \in$ $[0, c)$ in terms of $x \in\left[0, x_{*}(t ; c)\right)$, where

$$
x_{*}(t ; c)=c \log t+c \log \left(\frac{e^{2}}{2 c^{2}}\right),
$$

which is equivalent to $c(\log t)$ as $t \rightarrow \infty$. Then (1.4) is an even, continuous and nonnegative function defined for $x \in \mathbb{R}, t \geq c^{2} / 2$ and satisfying

$$
\begin{aligned}
& v_{*}(x, t ; c)=0 \text { for }|x| \geq x_{*}(t ; c), \\
& v_{*}(x, t ; c)>0 \text { for }|x|<x_{*}(t ; c),
\end{aligned}
$$


and

$$
\sup _{x \in \mathbb{R}} v_{*}(x, t ; c)=v_{*}(0, t ; c)=\frac{c^{2}}{2 t}<1 \quad \text { for } t>c^{2} / 2
$$

Going back to the variable $u$ by means of $(0.9)$, we get the explicit compactly supported solution

$$
u_{*}(x, t ; c)=-\frac{1}{\log v_{*}(x, t ; c)}
$$

of equation $(0.1),(0.2)$. Indeed, one can calculate from $(1.4),(1.5)$ that

$$
\exp \left\{-1 / u_{*}(x, t ; c)\right\}=\frac{1}{t}\left\{\frac{x_{*}(t)-|x|}{\left|\log \left(x_{*}(t)-|x|\right)\right|}\right\}(1+o(1))
$$

near the interfaces $x= \pm x_{*}(t ; c)$, and hence $\exp \left\{-1 / u_{*}\right\} \in C^{1}$, which implies the continuity of the heat flux on the interfaces. There holds [Ki]

$$
\left\|u_{*}(\cdot, t ; c)\right\|_{L^{1}(\mathbb{R})}=2 c \quad \text { for } \quad t \quad .2 / 2
$$

It is curious that at $t_{0}=c^{2} / 2$ the function $u_{*}\left(x, t_{\ldots}\right.$, , ehaves near $x=0$ like $|x|^{-2 / 3}$, which of course is an integrable singularity, but not a 0 -function.

We begin with some simple propertics of our explicit solution.

LEMMA 1. For any fixed $c>0$

$$
v_{*}(x, t ; c)=\frac{1}{t} F_{c}(\eta)+O\left(\frac{1}{t \log t}\right) \text { as } t \rightarrow \infty
$$

uniformly for $\eta \in \mathbb{R}$.

Proof. Using (1.5) yields

$$
|\eta|=w+\frac{\log \left(2 e^{2} t /(t+2)\right)}{\log (t+2)} w+\frac{(c-w) \log (c-w)-(c+w) \log (c+w)}{\log (t+2)}
$$

for $w \in(0, c)$. Hence,

$$
w(x, t ; c)=|\eta|+O(1 / \log t) \text { as } t \rightarrow \infty \text { in }\left\{|x| \leq x_{*}(t ; c)\right\}
$$

which by (1.4) completes the proof. $\square$ 
Lemma 2. For any fixed $0<c_{1}<c_{2}$ there holds

$$
\begin{gathered}
v_{*}\left(x, t ; c_{2}\right)>v_{*}\left(x, t ; c_{1}\right) \\
\text { for } x \in\left\{|x|<x_{*}\left(t ; c_{2}\right)\right\}, t>2 c_{2}^{2} .
\end{gathered}
$$

Proof. First we note that

$$
\frac{d}{d c} x_{*}(t ; c)>0 \text { for } t>2 c^{2}
$$

Using (1.4), we get

$$
\frac{d}{d c} v_{*}(x, t ; c)=\frac{1}{t}\left(c-w \cdot w_{c}^{\prime}\right)
$$

and (1.5) yields that $w_{c}^{\prime}(x, t ; c)$ is well defined in $\left\{|x|<x_{*}(t ; c)\right\}$ for $t>c^{2} / 2$. One can see that

$$
w_{c}^{\prime}=\frac{\log (c+w)-\log (c-w)}{\log (2 t)-\log (c-w)-\log (c+w)}<1
$$

for $w \in(0, c), t>2 c^{2}$. This together with (1.15) implies that $c-w \cdot w_{c}^{\prime}>0$ for $w \in(0, c)$, and hence by (1.15)

$$
\frac{d}{d c} v_{*}(x, t ; c)>0 \text { for } x \in\left\{|x|<x_{*}(t ; c)\right\}, \quad t>2 c^{2} .
$$

Using (1.14), (1.17), we get (1.13) completing the proof.

2. First Estimates. Let $u(x, t)$ be the solution of the problem (0.1)-(0.3). Assume now that $u_{0}$ has a compact support in an interval $[-b, b]$. We begin with an upper estimate of this solution.

LEMma 3. There exist constants $c_{1}>0$ and $t_{1}>c_{1}^{2} / 2$ such that

$$
v(x, t) \leq v_{*}\left(x, t_{1}+t ; c_{1}\right) \quad \text { in } \quad \mathbb{R} \times(0, \infty)
$$

Proof. By the comparison theorem [K] we obtain that (2.1) will be valid if

$$
v(x, 0) \leq v_{*}\left(x, t_{1} ; c_{1}\right) \quad \text { in } \quad \mathbb{R} .
$$

Using properties (1.6)-(1.9), we have that (2.2) holds if

$$
\begin{gathered}
t_{1}>c_{1}^{2} / 2 \\
\sup _{x \in \mathbb{R}} v_{*}\left(x, t_{1} ; c_{1}\right) \equiv \frac{c_{1}^{2}}{2 t_{1}}>\sup _{x \in \mathbb{R}} v(x, 0)=M_{1} \in(0,1)
\end{gathered}
$$


and

$$
\begin{gathered}
x_{*}\left(t_{1} ; c_{1}\right)=c_{1} \log \left(\frac{e^{2} t_{1}}{2 c_{1}^{2}}\right) \gg \\
l_{1}=\sup \{|x|: x \in \operatorname{supp} v(x, 0)\} .
\end{gathered}
$$

Choose $t_{1}$ as follows:

$$
t_{1}=\frac{1}{4} c_{1}^{2}\left(1+\frac{1}{M_{1}}\right)
$$

Then (2.3), (2.4) hold, and (2.5) implies the inequality

$$
c_{1} \log \left(\frac{e^{2}}{8}\left(1+\frac{1}{M_{1}}\right)\right) \gg l_{1}
$$

which is valid for any $c_{1}>0$ large enough.

Our next estimate is a lower bound.

LEMMA 4. There exist constants $c_{2}>0$ and $t_{2}>c_{2}^{2} / 2$ such that

$$
v(x, t) \geq v_{*}\left(x, t ; c_{2}\right) \quad \text { in } \quad \mathbb{R} \times\left(t_{2}, \infty\right)
$$

Proof. By well known properties of the weak solution $u(x, t)[\mathrm{K}]$ there exists $t_{2} \geq 0$ such that $u\left(0, t_{2}\right)>0$ and $u\left(x, t_{2}\right) \in C(\mathbb{R})$. Choose arbitrary small $c_{2}>0$. Then from (1.6)-(1.9) one can see that inequality

$$
v\left(x, t_{2}\right) \geq v_{*}\left(x, t_{2} ; c_{2}\right) \quad \text { in } \quad \mathbb{R}
$$

holds, and hence by the comparison theorem $[\mathrm{K}]$ estimate $(2.6)$ is valid.

If we now perform the change of variables (0.15), then from Lemmas 1,3 and 4 and properties (1.6)-(1.9) of the explicit solution we get the following weak form of the asymptotic behaviour, which in particular determines the rate of stabilization to 0 of $u(x, t)$.

Lemma 5. If $u_{0}$ satisfies (0.4) and has a compact support, then there exist $\tau_{*}>0$ and constants $0<c_{-}<c_{+}$such that

$$
F\left(\eta ; c_{-}\right) \leq \theta(\eta, \tau) \leq F\left(\eta ; c_{+}\right) \quad \text { in } \quad \mathbb{R} \times\left(\tau_{*}, \infty\right)
$$

As a consequence of these estimates we can also control the growth of the support of the solution $u(x, t)$ as $t \rightarrow \infty$. 
Corollary. There exist $t_{*}>0$ and $0<C_{-}<C_{+}$such that for $t \geq t_{*}$

$$
\begin{gathered}
\left\{|x|<C_{-} \log \left(\frac{e^{2} t}{2 C_{-}^{2}}\right)\right\} \subseteq \operatorname{supp} u(\cdot, t) \subseteq \\
\left\{|x|<C_{+} \log \left(\frac{e^{2} t}{2 C_{+}^{2}}\right)\right\}
\end{gathered}
$$

and

$$
-\left\{\log \frac{C_{-}^{2}}{2 t}\right\}^{-1} \leq \sup _{x \in \mathbb{R}} u(x, t) \leq-\left\{\log \frac{C_{+}^{2}}{2 t}\right\}^{-1} .
$$

3. A Sharp Estimate. We establish here a sharp lower bound.

Lemma 6. There holds

$$
\varliminf_{t \rightarrow \infty} t \sup _{x \in \mathbb{R}} v(x, t) \geq \frac{a^{2}}{2}
$$

where $a$ is given by $(0.8)$.

Proof. Step 1. Assume also that $u_{0}$ has a compact support. By well-known properties of the weak solution to the Cauchy problem $(0.1)-(0.3)$ there exists $t=t_{1}$ such that the support

$$
\operatorname{supp} v\left(x, t_{1}\right)=\left(l_{-}, l_{+}\right)
$$

is a connected interval and $0 \in\left(l_{-}, l_{+}\right)$. By Aleksandrov's Reflection Principle (see [GNN]) the solution $v(x, t)$ is a monotone function with respect to $x$ in $(-\infty,-b) \cup(b, \infty)$ for any fixed $t \geq t_{1}$.

Step 2. Fix now an arbitrary small $\varepsilon>0$. We replace $v\left(x, t_{1}\right)$ by an approximation $\tilde{v}_{\varepsilon}(x)$ such that

i) $\tilde{v}_{\varepsilon}(x) \leq v\left(x, t_{1}\right)$ in $\mathbb{R}$ and $\tilde{v}_{\varepsilon}(x) \equiv v\left(x, t_{1}\right)$ in $\left(l_{-}+\varepsilon, l_{+}-\varepsilon\right)$,

ii) $\int\left(u\left(x, t_{1}\right)-\tilde{u}_{\varepsilon}(x)\right) d x \leq 2 \varepsilon$, and

iii) $\left|\frac{d}{d x} \tilde{v}_{\varepsilon}(x)\right| \geq 1$ near the endpoints of its support.

Construction. Consider the right-hand side $x>0$. It is clear that we can choose $l_{1} \in\left(l_{+}-\varepsilon / 2, l_{+}\right)$such that

$$
\int_{l_{1}}^{l_{+}} u\left(x, t_{1}\right) d x<\varepsilon / 2 .
$$


To the left of $l_{1}$ we draw the line $y(x)=M\left(l_{1}-x\right)$. This line intersects the graph of $v\left(x, t_{1}\right)$ for the first time in a point $l_{2}<l_{1}$. If $M>1$ is large enough, we have $l_{2}>l_{+}-\varepsilon$ and

$$
\int_{l_{2}}^{l_{+}} u\left(x, t_{1}\right) d x<\varepsilon
$$

For such an $M$ we define

$$
\begin{aligned}
& \tilde{v}_{\varepsilon}(x)=v\left(x, t_{1}\right) \quad \text { if } \quad 0 \leq x \leq l_{2}, \\
& \tilde{v}_{\varepsilon}(x)=y(x) \quad \text { if } \quad l_{2} \leq x \leq l_{1}, \\
& \tilde{v}_{\varepsilon}(x)=0 \quad \text { if } \quad x \geq l_{1} .
\end{aligned}
$$

The same construction holds for the left-hand side $x<0$.

Step 3. Denote $v_{\varepsilon}(x, t)$ the weak solution of the Cauchy problem in $\mathbb{R} \times\left(t_{1}, \infty\right)$ to the equation $(0.6)$ with the initial function $v_{\varepsilon}\left(x, t_{1}\right)=\tilde{v}_{\varepsilon}(x)$ in $\mathbb{R}$. Let

$$
c_{\varepsilon}=\frac{1}{2} \int \tilde{u}_{\varepsilon}(x) d x \equiv \frac{1}{2} \int u_{\varepsilon}(x, t) d x \text { for every } t \geq t_{1},
$$

so that $a-\varepsilon \leq c_{\varepsilon}<a$. Since by construction $\tilde{v}_{\varepsilon}(x) \leq v\left(x, t_{1}\right)$ in $\mathbb{R}$, from the comparison theorem $[\mathrm{K}]$ we have $v_{\varepsilon}(x, t) \leq v(x, t)$ in $\mathbb{R} \times\left(t_{1}, \infty\right)$.

We consider now the family of explicit solutions $\left\{v_{*}\left(x-x_{0}, t+T ; c_{\varepsilon}\right), x_{0} \in[-b, b], T>0\right\}$ having the same mass $c_{\varepsilon}$ as $u_{\varepsilon}(x, t)$. For a fixed $t \geq t_{1}$ we denote by $N\left(t ; x_{0}, T\right)$ the number of sign changes in $\mathbb{R}$ of the difference $w\left(x, t ; x_{0}, T\right) \equiv v_{\varepsilon}(x, t)-v_{*}\left(x-x_{0}, t+T ; c_{\varepsilon}\right)$ or, which is the same, the number of intersections in $\mathbb{P}$ of the functions $v_{\varepsilon}(x, t)$ and $v_{*}\left(x-x_{0}, t+T ; c_{\varepsilon}\right)$. Then by a well-known property, see e.g. [A], [GP1], [M], [Sat] and references therein, we conclude that $N\left(t ; x_{0}, T\right)$ does not increase with time and, in particular,

$$
N\left(t ; x_{0}, T\right) \leq N\left(t_{1} ; x_{0}, T\right) \quad \text { for } \quad t>t_{1} .
$$

Notice that by known properties of regularity of the weak solution at a point where it is positive $[\mathrm{K}]$, and by using results of $[\mathrm{A}],[\mathrm{KP}]$, we may conclude that for $t \geq t_{1}$ every zero of the difference in the domain of positiveness of both solutions considered is an isolated point. Since, by the properties of the explicit solutions given in Section 1, we have for an arbitrary fixed $x_{0} \in[-b, b]$

$$
v_{*}\left(x-x_{0}, t_{1}+T ; c_{\varepsilon}\right) \approx \frac{c_{\varepsilon}^{2}}{2\left(t_{1}+T\right)} \text { as } T \rightarrow \infty
$$

uniformly in any compact in $x \in \mathbb{R}$, by using the property iii) of the function $v_{\varepsilon}\left(x, t_{1}\right)$, we have that for every $x_{0} \in[-b, b]$ and $T$ large enough

$$
N\left(t_{1} ; x_{0}, T\right)=2 \text {. }
$$


This together with (3.3) yields the inequality

$$
N\left(t ; x_{0}, T\right) \leq 2 \text { for } t \geq t_{1}
$$

Fix arbitrary $x_{0} \in[-b, b]$ and $T=T_{0}$ large enough. We now prove that for $t>t_{1}$

$$
\sup _{x \in \mathbb{R}} v_{\varepsilon}(x, t) \geq \sup _{x \in \mathbb{R}} v_{*}\left(x-x_{0}, t+T_{0} ; c_{\varepsilon}\right)
$$

Assume for a moment that this is true. Then

$$
\sup _{x \in \mathbb{R}} v_{*}\left(x-x_{0}, t+T_{0} ; c_{\varepsilon}\right) \equiv v_{*}\left(0, t+T_{0} ; c_{\varepsilon}\right)=\frac{c_{\varepsilon}^{2}}{2\left(t+T_{0}\right)}
$$

and (3.6) implies that

$$
\varliminf_{t \rightarrow \infty} t \sup _{x \in \mathbb{R}} v(x, t) \geq \varliminf_{t \rightarrow \infty} t \sup _{x \in \mathbb{R}} v_{\varepsilon}(x, t) \geq \frac{c_{\varepsilon}^{2}}{2}
$$

Since $\varepsilon>0$ is an arbitrary, we obtain the desired result (3.1). Hence, we have to prove (3.6).

Step 4. Suppose (3.6) is not valid and

$$
t_{*}=\sup \left\{\tau>0:(3.6) \text { holds for all } t \in\left[t_{1}, t_{1}+\tau\right]\right\}<\infty
$$

Let $x_{*} \in[-b, b]$ be a point of maximum of the function $v_{\varepsilon}\left(x, t_{*}\right)$ and hence by a definition of $t_{*}$ we have

$$
v_{\varepsilon}\left(x_{*}, t_{*}\right)=\frac{c_{\varepsilon}^{2}}{2\left(t_{*}+T_{0}\right)}
$$

Consider the explicit solution $v_{*}\left(x-x_{*}, t+T_{0} ; c_{\varepsilon}\right)$. By construction we have

$$
\begin{gathered}
w\left(x, t_{*} ; x_{*}, T_{0}\right) \equiv v_{\varepsilon}\left(x, t_{*}\right)-v_{*}\left(x-x_{*}, t_{*}+T_{0} ; c_{\varepsilon}\right)=0 \quad \text { for } \quad x=x_{*}, \\
w_{x}\left(x, t_{*} ; x_{*}, T_{0}\right)=0 \quad \text { for } \quad x=x_{*}
\end{gathered}
$$

Suppose first that $x=x_{*}$ is the point of tangency of the functions $v_{\varepsilon}\left(x, t_{*}\right)$ and $v_{*}(x-$ $\left.x_{*}, t_{*}+T_{0} ; c_{\varepsilon}\right)$, i.e., the difference $w\left(x, t_{*} ; x_{*}, T_{0}\right)$ satisfying $(3.8)$ does not change sign in a small neighborhood of the point $x=x_{*}$. Since these have the same masses we get

$$
N\left(t_{*} ; x_{*}, T_{0}\right) \geq 1
$$

Indeed, if (3.9) is not valid and $N\left(t_{*} ; x_{*}, T_{0}\right)=0$, then by the Strong Maximum Principle [Fr] it follows that, since $v_{\varepsilon} \not \equiv v_{*}$, for arbitrarily small $\delta>0$ either $v_{\varepsilon}\left(x, t_{*}+\delta\right)<v_{*}(x-$ 
$\left.x_{*}, t_{*}+T_{0}+\delta ; c_{\varepsilon}\right)$ or $v_{\varepsilon}\left(x, t_{*}+\delta\right)>v_{*}\left(x-x_{*}, t_{*}+T_{0}+\delta ; c_{\varepsilon}\right)$ in the domain of positiveness of both functions, contradicting the equality of masses. Hence, there exists at least one point of intersection, i.e., a point $x_{1}$ where the difference $w$ changes sign, and $x_{1} \neq x_{*}$. Assume without loss of generality that the difference $w\left(x, t_{*} ; x_{*}, T_{0}\right) \leq 0$ in a small neighborhood $I_{r}=\left(x_{*}-r, x_{*}+r\right)$ of the point $x=x_{*}$ with $r \ll\left|x_{1}-x_{*}\right|$. Then by using the continuous dependence of the function $v_{*}\left(x-x_{*}, t_{*}+T_{0} ; c_{\varepsilon}\right)$ with respect to a small perturbation of the value of $T_{0}$, we obtain that for any small $\delta>0$ there exist at least two points of sign change for perturbed difference $w\left(x, t_{*} ; x_{*}, T_{0}+\delta\right)$ in $I_{r}$, one to the left of $x=x_{*}$ and to the right, and also an intersection point which lies not far from $x_{1}$, and anyway is outside $I_{r}$. Therefore, for small $\delta>0$ we have

$$
N\left(t_{*} ; x_{*}, T_{0}+\delta\right) \geq 3
$$

This leads to a contradiction with (3.5) for $t=t_{*}, x_{0}=x_{*} \in[-b, b]$ and $T=T_{0}+\delta$.

Now, if the maximum $x=x_{*}$ is an inflection point for the difference $w\left(x, t_{*} ; x_{*}, T\right)$ satisfying (3.8), namely that it changes sign in any neighborhood of the point $x=x_{*}$, then we use the idea given in [GP1], [GP2]. Assume without loss of generality that $w\left(x, t_{*} ; x_{*}, T_{0}\right)>0$ in a small left-hand neighborhood of $x=x_{*}$ and $w\left(x, t_{*} ; x_{*}, T_{0}\right)<0$ in a small right-hand one. Then it is easily seen that for any $\lambda>0$ small enough there holds

$$
N\left(t_{*} ; x_{*}-\lambda, T_{0}\right) \geq 3
$$

(see a similar detailed analysis in [GP1], [GP2]) contradicting (3.5) for $t=t_{*}, x_{0}=x_{*}-\lambda$ and $T=T_{0}$ and completing the proof of Lemma 6 in the case of compactly supported data.

Step 5. If $u_{0}$ is not compactly supported, the proof is made by approximation from below with compactly supported functions.

4. Semiconvexity. For the proof of Theorem 1 we need also the following lower estimate of the second derivative of the solution.

Lemma 7. Let $u(x, t)$ be a solution of (0.1)-(0.3). Then for every $\varepsilon>0$ there exists $T_{\varepsilon}>0$ such that

$$
v_{x x} \geq-\frac{1+\varepsilon}{t(\log t)^{2}}
$$

in the domain $\left\{(x, t): x \in \mathbb{R}, t \geq T_{\varepsilon}\right\}$.

Proof. By approximation we may assume that $u_{0}$ is continuous, bounded and positive in $\mathbb{R}$. Then $v(x, t)$ is a classical solution of equation (0.6). Differentiating it twice with respect to $x$ we obtain the equation satisfied by $z=v_{x x}$ :

$$
\begin{gathered}
z_{t}=v(\log v)^{2} z_{x x}+2\left[(\log v)^{2}+2 \log v\right] v_{x} z_{x}+ \\
\frac{2}{v}(\log v+1) v_{x}^{2} z+\left((\log v)^{2}+2 \log v\right) z^{2} .
\end{gathered}
$$


Following the method of $[\mathrm{AB}]$ we want to try an explicit subsolution for this equation. If we try $z=-1 / \varphi(t)$, with $\varphi>0$, we easily check that a sufficient condition is that

$$
\varphi^{\prime}(t) \leq \inf _{x \in \mathbb{R}}\left((\log v(x, t))^{2}+2 \log v(x, t)\right)
$$

Now, for large $t>0$ from Lemma 5 we have $v \leq$ const $/ t$, hence $(\log v)^{2}+2 \log v \geq$ $(\log t)^{2}\left(1-\frac{\varepsilon}{4}\right)$ for $\varepsilon$ small if $t$ is large enough. Therefore, an admissible choice is

$$
\varphi(t)=\left(1-\frac{\varepsilon}{2}\right)(t-T)(\log t)^{2}
$$

if $t>T$ for some large $T$. Since with this choice $z$ will be a subsolution of equation (4.2) in $D=\{(x, t): x \in \mathbb{R}, t>T\}$ with $z(x, T)=-\infty$, we conclude from the Maximum Principle that

$$
v_{x x} \geq z \text { in } D
$$

hence the conclusion in the limit.

Remark. The sharpness of estimate (4.1) is checked by looking at the explicit solution $v_{*}(x, t ; c)$, for which we have the estimates

$$
\begin{gathered}
\left(v_{*}\right)_{x x} \geq-\frac{1}{t(\log t)^{2}}+O\left(\frac{1}{t(\log t)^{3}}\right) \quad \text { as } \quad t \rightarrow \infty \\
\left(v_{*}\right)_{x x}(0, t ; c)=-\frac{1}{t \log ^{2}\left(2 t / c^{2}\right)} \quad \text { for } \quad t>c^{2} / 2
\end{gathered}
$$

5. Proof of Theorem 2. We now prove, under the additional assumption that $u_{0}$ has a compact support, explicit estimates of the support of the solution

$$
\operatorname{supp} u(x, t)=\left(s_{-}(t), s_{+}(t)\right),
$$

which is a connected interval for large $t$, say $t>t_{1}$.

We take the function $U(x, t)=u_{*}\left(x-d_{+}, t+T ; a\right)$, where $a$ is one half of the energy of $u_{0}, T>a^{2} / 2$ and $d_{+}=s_{+}\left(t_{1}\right)+x_{*}(T ; a)$, so that the support of $U(x, 0),\left(S_{-}(0), S_{+}(0)\right)$, lies to the right of the support of $u_{0}$. Then, by the shifting comparison principle [V] (see similar results for quasilinear heat equation with source proved by intersection comparison in [SGKM, p. 248]) we have a comparison of the interfaces of $u$ and $U$, i.e., for $t>t_{1}$

$$
\begin{aligned}
& s_{+}(t) \leq S_{+}(t)=d_{+}+x_{*}(t+T ; a) \\
& s_{-}(t) \leq S_{-}(t)=d_{+}-x_{*}(t+T ; a) .
\end{aligned}
$$


A similar argument by shifting to the left gives

$$
\begin{aligned}
& s_{+}(t) \geq-d_{-}+x_{*}(t+T ; a), \\
& s_{-}(t) \geq-d_{-}-x_{*}(t+T ; a),
\end{aligned}
$$

where $d_{-}=s_{-}\left(t_{1}\right)-x_{*}(T ; a)$. In view of the formula for $x_{*}(t+T ; a)$ we have

$$
\begin{aligned}
& s_{+}(t)=a \log t+O(1), \\
& s_{-}(t)=-a \log t+O(1),
\end{aligned}
$$

which completes the proof.

6. Proof of Theorem 1. Consider the Cauchy problem $(0.16),(0.17)$ for the quasilinear parabolic equation which is a perturbation of the autonomous ("stationary") equation

$$
\theta_{\tau}=\mathcal{A}(\theta)
$$

By Lemma 5 the evolution trajectory $\left\{\theta(\cdot, \tau), \tau>\tau_{*}\right\}$ is uniformly bounded, and hence, by a general regularity result $[\mathrm{DB} 1],[\mathrm{DB} 2]$, it is compact in $C_{0}(\mathbb{R})$. We now prove that $\omega$-limit set, given by the formula

$$
\begin{gathered}
\omega\left(\theta_{0}\right)=\left\{f \in C_{0}(\mathbb{R}): f \geq 0 \text { and there exists }\left\{\tau_{j}\right\} \rightarrow \infty\right. \text { such that } \\
\left.\theta\left(\cdot, \tau_{j}\right) \rightarrow f(\cdot) \text { as } j \rightarrow \infty \text { uniformly in } \mathbb{R}\right\}
\end{gathered}
$$

is precisely

$$
\omega\left(\theta_{0}\right)=\left\{F_{a}(\cdot)\right\},
$$

which indeed yields (0.7).

Choose an arbitrary $f \in \omega\left(\theta_{0}\right)$, so that there exists a sequence $\tau_{j} \rightarrow \infty$ such that

$$
\theta\left(\cdot, \tau_{j}\right) \rightarrow f(\cdot) \text { as } j \rightarrow \infty \text { uniformly in } \mathbb{R} .
$$

Applying Aleksandrov's Reflection Principle and passing to the limit $\tau \rightarrow \infty$ we have $f=f(|\eta|)$ and $f$ does not increase in $|\eta|$ (see a detailed argument in [KV], Section 5).

We now prove that $f(a)=0$. Suppose for a contradiction that $f(a)>0$ and hence by continuity

$$
\text { messupp } f>2 a \text {. }
$$


Using the conservation law (1.3) for the rescaled function $\theta$ with $\tau=\tau_{j}$ yields

$$
\int_{-\infty}^{+\infty}\left[1-\frac{\log \theta\left(\eta, \tau_{j}\right)}{\log \tau_{j}}\right]^{-1} d \eta \equiv E_{0}=2 a
$$

for $j=1,2, \ldots$ It follows from (6.3) that for given small $\varepsilon>0$ there exists $j_{\varepsilon}>0$ such that

$$
\theta\left(\cdot, \tau_{j}\right) \geq(f(\cdot)-\varepsilon)_{+} \text {in } \mathbb{R} \text { for any } j>j_{\varepsilon}
$$

Therefore (6.4), (6.6) imply that for any $\varepsilon>0$ small enough

$$
\operatorname{mes} \operatorname{supp} \theta\left(\cdot, \tau_{j}\right) \geq \operatorname{mes} \operatorname{supp}(f(\cdot)-\varepsilon)_{+}>2 a
$$

for $j>j_{\varepsilon}$. Combining (6.5)-(6.7) yields the estimate

$$
\begin{gathered}
\int_{-\infty}^{+\infty}\left[1-\frac{\log \theta\left(\eta, \tau_{j}\right)}{\log \tau_{j}}\right]^{-1} d \eta \geq \int_{-\infty}^{+\infty}\left[1-\frac{\log \left((f(\eta)-\varepsilon)_{+}\right)}{\log \tau_{j}}\right]^{-1} d \eta \\
\rightarrow \operatorname{mes} \operatorname{supp}(f(\cdot)-\varepsilon)_{+}>2 a \text { as } j \rightarrow \infty,
\end{gathered}
$$

contradicting the conservation law (6.5).

Thus, $f(a)=0$ and mes supp $f \leq 2 a$. Using Lemma 6 yields

$$
f(0) \geq \frac{a^{2}}{2}
$$

Rewriting estimate (4.1) for the function $\theta(\eta, \tau)$, integrating this inequality twice and passing to the limit $\tau=\tau_{j} \rightarrow \infty$, we obtain

$$
f(\eta) \geq \frac{1}{2}\left(f(0)-\eta^{2}\right)_{+}
$$

By using (4.1), we may also conclude that $f_{\eta \eta} \geq-1$ a.e. Since $F_{\eta \eta}(\eta ; a) \equiv-1$ in $[0, a]$, from (6.9) and (6.10) we have that the difference $z(\eta)=f(\eta)-F_{a}(\eta)$ satisfies $z \geq 0, z_{\eta \eta} \geq 0$ a.e. in $[0, a]$, and since $z(a)=0$, one can see that $z_{\eta}(a) \leq 0$. Assume for contradiction that $z \not \equiv 0$ and hence $z_{\eta \eta}>0$ in a set of nonzero measure in $[0, a]$. Then integrating the inequality $z_{\eta \eta} \geq 0$ over $(0, a)$ yields

$$
z_{\eta}(0)<z_{\eta}(a) \leq 0
$$

contradicting the symmetry condition at the origin. This completes the proof. 
7. Final Remarks. It is interesting to consider our equation $(0.1),(0.2)$ as some kind of limit of the porous medium equation (PME) $u_{t}=\left(u^{m}\right)_{x x}$ as $m \rightarrow \infty$. Thus, the PME admits a family of explicit self-similar solutions (the Barenblatt solutions) which decay in time according to

$$
u_{m}(x, t) \leq a_{m}\left\|u_{0}\right\|_{1}^{\frac{2}{m+1}} t^{-\frac{1}{m+1}}, a_{m}>0
$$

while their support is confined by the interfaces $\pm s_{m}(t)$,

$$
s_{m}(t)=c_{m}\left\|u_{0}\right\|_{1}^{\frac{m-1}{m+1}} t^{\frac{1}{m+1}}, c_{m}>0
$$

Moreover, these estimates are true for every nonnegative solution while initial data are compactly supported [V]. Put now $v=u^{m}$ (in analogy to (0.5)) and let $m \rightarrow \infty$ to obtain a formal expression for the upper bound in the limit

$$
v_{\infty}(x, t) \leq a_{\infty}\left\|u_{0}\right\|_{1}^{2} t^{-1}
$$

which agrees with Theorem 1. Agreement with Theorem 2 for the interfaces necessitates replacing $t^{1 /(m+1)}$ in the limit by $\log t$ (and not by 1 ), then obtaining

$$
s_{\infty}(t)=c_{\infty}\left\|u_{0}\right\|_{1} \log t .
$$

On the other hand, a rigorous limit $m \rightarrow \infty$ in the equation $u_{t}=\left(\phi_{m}(u)\right)_{x x}$ leads to the so called "mesa problem" studied by several authors, cf. [CF], [EHKO], [FH], [Sac].

Acknowledgement. The first author is grateful to the Department of Mathematics and the University Autónoma of Madrid for their hospitality during his visit to Madrid to work on this project. The second author thanks the Keldysh Institute of Applied Mathematics of the USSR Academy of Sciences for support of the visit to Moscow. These visits have been supported by grant OIVTA AN SSSR "Math. Modeling Theory Nonl. Phenom. Synergetics" and by EEC Contract SC1-0019-C. The work was finished during a visit of both authors to the IMA to participate in the Program on Phase Transitions and Degenerate Diffusions. The authors are grateful to Dr. Andrzey Herczynski for drawing their attention to the paper [Ki].

\section{REFERENCES}

[A] S. ANGENENT, The zero set of a solution of a parabolic equation, J. reine angew. Math. 390 (1988), pp. $79-96$.

[AB] D.G. Aronson \& P. BÉnilan, Régularité des solutions de l'equation des milieux poreux dan $\mathbb{R}^{n}$, Comptes Rendus Acad. Sci. Paris, Ser. 1, 288 (1979), pp. 103-105.

[BMfF] J.R. Burgan, A. Munier, M.R. Feix \& E. Fijalkow, Homology and the nonlinear heat differential equation, SIAM Jour. Appl. Math. 44 (1984), pp. 11-18. 
[CF] L.A. Caffarelli \& A. Friedman, Asymptotic behavior of solutions of $u_{t}=\Delta u^{m}$ as $m \rightarrow \infty$, Indiana Univ. Math. Jour. 36 (1987), pp. 711-718.

[DB1] E. Di Benedetto, Continuity of weak solutions to a general porous medium equation, Indiana Univ. Math. Jour. 32 (1983), pp. 83-119.

[DB2] E. Di BenedetTo, A boundary modulus of continuity of a class of singular parabolic equations, Jour. Diff. Equations 63 (1986), pp. 418-447.

[EHKo] C.M. Elliot, M.A. Herrero, J.R. King \& J.R. Ockendon, The mesa problem: diffusion patterns for $u_{t}=\nabla\left(u^{m} \nabla u\right)$ as $m \rightarrow \infty$, IMA Jour. Appl. Math. 37 (1986), pp. 147-154.

[F] G. Francsics, The porous medium equation: the superslow diffusion case, Nonlinear Analysis TMA 12 (1988), pp. 291-301.

[Fr] A. Friedman, Partial Differential Equations of Parabolic Type, Englewood Cliffs, Prentice Hall, NJ, 1964.

[FH] A. Friedman \& S. Huang, Asymptotic behavior of solutions of $u_{t}=\Delta \phi_{m}(u)$ as $m \rightarrow \infty$ with inconsistent initial values, Analyse Mathématique et Applications, Gauthier-Villars, Paris. (1988), pp. $165-180$.

[GKV] V.A. Galaktionov, R. Kersner \& J.L. Vazquez, Asymptotic behaviour for an equation of superslow diffusion in a bounded domain, to appear.

[GP1] V.A. Galaktionov \& S.A. Posashkov, Applications of a new comparison theorems for unbounded solutions of a nonlinear parabolic equations, Differentsial'nye Uravneniya 22 (1986), pp. 1165-1173 (in Russian).

[GP2] V.A. Galaktionov \& S.A. Posashkov, Any large solution of a non-linear heat conduction equation becomes monotonic in time, Proc. Royal Soc. Edinburgh 118A (1991).

[GV] V.A. Galaktionov \& J.L. Vazquez, Asymptotic behaviour of nonlinear parabolic equations with critical exponents. A dynamical system approach, to appear.

[GNN] B. Gidas, W.-M. Ni \& L. Nirenberg, Symmetry and related properties via the maximum principle, Comm. Math. Phys. 68 (1979), pp. 209-243.

[K] A.S. Kalashnikov, Some questions on the qualitative theory of nonlinear degenerate parabolic second-order equations, Uspekhi Matem. Nauk 42 (1987), pp. 135-176 (in Russian).

[Ki] J.R. KING, Exact solutions to some nonlinear diffusion equations, Quart. Jour. Mech. Appl. Math. 42 (1989), pp. 537-552.

[KP] K. Kunisch \& G. PEICHL, On the shape of the solutions of second order parabolic differential equations, Jour. Diff. Equations 75 (1988), pp. 329-353.

[KV] S. Kamin \& J.L. Vazquez, Fundamental solutions and asymptotic behaviour for the p-laplacian equation, Revista Matemática Iberoamericana 4 (1988), pp. 339-354.

[M] H. Matano, Nonincrease of the lap number for a one-dimensional semi-linear parabolic equation, Jour. Fac. Sci. Univ. Tokyo, Sec: IA, 29 (1982), pp. 401-441.

[SGKM] A.A. Samarskit, V.A. Galaktionov, S.P. Kurdyumov \& A.P. Mikhailov, Blow-up in Problems for Quasilinear Parabolic Equations, Nauka, Moscow, 1987 (English translation: Walter de Gruyter, Berlin, to appear).

[Sac] P.E. SACKs, A singular limit for the porous medium equation, Jour. Math. Anal. Appl. 140 (1989), pp. $456-466$.

[Sat] D.H. Sattinger, On the total variation of solutions of parabolic equations, Math. Ann. 183 (1969), pp. 78-92.

[V] J.L. VAzquez, Asymptotic behaviour and propagation properties of the one-dimensional flow of a gas in a porous medium, Trans. Amer. Math. Soc. 277 (1983), pp. 507-527. 
L.A. Peletier \& W.C. Troy, Self-similar solutions for infiltration of dopant into semiconductors

H. Scott Dumas and James A. Ellison, Nekhoroshev's theorem, ergodicity, and the motion of energetic charged particles in crystals

Stathis Filippas and Robert V. Kohn, Refined asymptotics for the blowup of $u_{t}-\Delta u=u^{p}$.

Patricia Bauman, Nicholas C. Owen and Daniel Phillips, Maximum principles and a priori estimates for an incompressible material in nonlinear elasticity

Patricia Bauman, Nicholas C. Owen and Daniel Phillips, Maximal smoothness of solutions to certain Euler-Lagrange equations from nonlinear elasticity

Jack Carr and Robert Pego, Self-símilarity in a coarsening model in one dimension

J.M. Greenberg, The shock generation problem for a discrete gas with short range repulsive forces

George R. Sell and Mario Taboada, Local dissipativity and attractors for the Kuramoto-Sivashinsky equation in thin $2 \mathrm{D}$ domains

T. Subba Rao, Analysis of nonlinear time series (and chaos) by bispectral methods

Nicholas Baumann, Daniel D. Joseph, Paul Mohr and Yuriko Renardy, Vortex rings of one fluid in another free fall

Oscar Bruno, Avner Friedman and Fernando Reitich, Asymptotic behavior for a coalescence problem

Johannes C.C. Nitsche, Periodic surfaces which are extremal for energy functionals containing curvature functions

F. Abergel and J.L. Bona, A mathematical theory for viscous, free-surface flows over a perturbed plane Gunduz Caginalp and Xinfu Chen, Phase field equations in the singular limit of sharp interface problems Robert P. Gilbert and Yongzhi Xu, An inverse problem for harmonic acoustics in stratified oceans Roger Fosdick and Eric Volkmann, Normality and convexity of the yield surface in nonlinear plasticity H.S. Brown, I.G. Kevrekidis and M.S. Jolly, A minimal model for spatio-temporal patterns in thin film flow Chao-Nien Chen, On the uniqueness of solutions of some second order differential equations

Xinfu Chen and Avner Friedman, The thermistor problem for conductivity which vanishes at large temperature Xinfu Chen and Avner Friedman, The thermistor problem with one-zero conductivity

E.G. Kalnins and W. Miller, Jr., Separation of variables for the Dirac equation in Kerr Newman space time E. Knobloch, M.R.E. Proctor and N.O. Weiss, Finite-dimensional description of doubly diffusive convection V.V. Pukhnachov, Mathematical model of natural convection under low gravity

M.C. Knaap, Existence and non-existence for quasi-linear elliptic equations with the p-laplacian involving critical Sobolev exponents

Stathis Filippas and Wenxiong Liu, On the blowup of multidimensional semilinear heat equations
A.M. Meirmanov, The Stefan problem with surface tension in the three dimensional case with spherical symmetry: non-existence of the classical solution

Bo Guan and Joel Spruck, Interior gradient estimates for solutions of prescribed curvature equations of parabolic type

Hi Jun Choe, Regularity for solutions of nonlinear variational inequalities with gradient constraints

Peter Shi and Yongzhi Xu, Quasistatic linear thermoelasticity on the unit disk

Satyanad Kichenassamy and Peter J. Olver, Existence and non-existence of solitary wave solutions to higher order model evolution equations

Dening Li, Regularity of solutions for a two-phase degenerate Stefan Problem

Marek Fila, Bernhard Kawohl and Howard A. Levine, Quenching for quasilinear equations

Yoshikazu Giga, Shun'ichi Goto and Hitoshi Ishii, Global existence of weak solutions for interface equations coupled with diffusion equations

Mark J. Friedman and Eusebius J. Doedel, Computational methods for global analysis of homoclinic and hetero clinic orbits: a case study

Mark J. Friedman, Numerical analysis and accurate computation of heteroclinic orbits in the case of center manifolds

Peter W. Bates and Songmu Zheng, Inertial manifolds and inertial sets for the phase-field equations

J. López Gómez, V. Márquez and N. Wolanski, Global behavior of positive solutions to a semilinear equation with a nonlinear flux condition

Xinfu Chen and Fahuai Yi, Regularity of the free boundary of a continuous casting problem
Eden, A., Foias, C., Nicolaenko, B. and Temam, R., Inertial sets for dissipative evolution equations Part I: Construction and applications

813 Jose-Francisco Rodrigues and Boris Zaltzman, On classical solutions of the two-phase steady-state Stefan problem in strips

814 Viorel Barbu and Srdjan Stojanovic, Controlling the free boundary of elliptic variational inequalities on a variable domain

Viorel Barbu and Srdjan Stojanovic, A variational approach to a free boundary problem arising in electrophotography 
Shoshana Kamin, Lambertus A. Peletier and Juan Luis Vazquez, On the Barenblatt equation of elastoplastic filtration

Avner Friedman and Bei $\mathbf{H u}$, The Stefan problem with kinetic condition at the free boundary

M.A. Grinfeld, The stress driven instabilities in crystals: mathematical models and physical manifestations

Bei Hu and Lihe Wang, A free boundary problem arising in electrophotography: solutions with connected toner region

\section{Yongzhi Xu, T. Craig Poling, and Trent Brundage, Direct and inverse scattering of time harmonic} acoustic waves in an inhomogeneous shallow ocean

Steven J. Altschuler, Singularities of the curve shrinking flow for space curves

Steven J. Altschuler and Matthew A. Grayson, Shortening space curves and flow through singularities

Tong Li, On the Riemann problem of a combustion model

L.A. Peletier \& W.C. Troy, Self-similar solutions for diffusion in semiconductors

C.J. van Duijn, L.A. Peletier \& R.J. Schotting, On the analysis of brine transport in porous media

Minkyu Kwak, Finite dimensional description of convective reaction-diffusion equations

Minkyu Kwak, Finite dimensional inertial forms for the 2D Navier-Stokes equations

Victor A. Galaktionov and Sergey A. Posashkov, On some monotonicity in time properties for a quasilinear parabolic equation with source

Victor A. Galaktionov, Remark on the fast diffusion equation in a ball

Hi Jun Choe and Lihe Wang, A regularity theory for degenerate vector valued variational inequalities

Vladimir I. Oliker and Nina N. Uraltseva, Evolution of nonparametric surfaces with speed depending on curvature, II. The mean curvature case.

S. Kamin and W. Liu, Large time behavior of a nonlinear diffusion equation with a source

Shoshana Kamin and Juan Luis Vazquez, Singular solutions of some nonlinear parabolic equations

Bernhard Kawohl and Robert Kersner, On degenerate diffusion with very strong absorption

Avner Friedman and Fernandor Reitich, Parameter identification in reaction-diffusion models

E.G. Kalnins, H.L. Manocha and Willard Miller, Jr., Models of $q$-algebra representations I. Tensor products of special unitary and oscillator algebras

Robert J. Sacker and George R. Sell, Dichotomies for linear evolutionary equations in Banach spaces

Oscar P. Bruno and Fernando Reitich, Numerical solution of diffraction problems: a method of variation of boundaries

Oscar P. Bruno and Fernando Reitich, Solution of a boundary value problem for Helmholtz equation via variation of the boundary into the complex domain

Victor A. Galaktionov and Juan L. Vazquez, Asymptotic behaviour for an equation of superslow diffusion. The Cauchy problem

Josephus Hulshof and Juan Luis Vazquez, The Dipole solution for the porous medium equation in several space dimensions

Shoshana Kamin and Juan Luis Vazquez, The propagation of turbulent bursts

Miguel Escobedo, Juan Luis Vazquez and Enrike Zuazua, Source-type solutions and asymptotic behaviour for a diffusion-convection equation

Marco Biroli and Umberto Mosco, Discontinuous media and Dirichlet forms of diffusion type

Stathis Filippas and Jong-Shenq Guo, Quenching profiles for one-dimensional semilinear heat equations

H. Scott Dumas, A Nekhoroshev-like theory of classical particle channeling in perfect crystals

R. Natalini and A. Tesei, On a class of perturbed conservation laws

Paul K. Newton and Shinya Watanabe, The geometry of nonlinear Schrödinger standing waves

S.S. Sritharan, On the nonsmooth verification technique for the dynamic programming of viscous flow

Mario Taboada and Yuncheng You, Global attractor, inertial manifolds and stabilization of nonlinear damped beam equations

Shigeru Sakaguchi, Critical points of solutions to the obstacle problem in the plane

F. Abergel, D. Hilhorst and F. Issard-Roch, On a dissolution-growth problem with surface tension in the neighborhood of a stationary solution

Erasmus Langer, Numerical simulation of MOS transistors

Haïm Brezis and Shoshana Kamin, Sublinear elliptic equations in $\mathbb{R}^{n}$

Johannes C.C. Nitsche, Boundary value problems for variational integrals involving surface curvatures

Chao-Nien Chen, Multiple solutions for a semilinear elliptic equation on $\mathbb{R}^{N}$ with nonlinear dependence on the gradient

D. Brochet, X. Chen and D. Hilhorst, Finite dimensional exponential attractor for the phase field model

Joseph D. Fehribach, Mullins-Sekerka stability analysis for melting-freezing waves in helium-4

Walter Schempp, Quantum holography and neurocomputer architectures

D.V. Anosov, An introduction to Hilbert's 21st problem

Herbert E Huppert and M Grae Worster, Vigorous motions in magma chambers and lava lakes 